\title{
Effect of the reporting rate of synchrophasor measurements for distributed secondary control of AC microgrid
}

\author{
Edoardo De Din, Gianluca Lipari, Andrea Angioni, Ferdinanda Ponci, Antonello Monti \\ Institute for Automation of Complex Power Systems \\ E.ON ERC - RWTH Aachen University \\ Aachen, Germany \\ [ededin,glipari,aangioni,fponci,amonti]@eonerc.rwth-aachen.de
}

\begin{abstract}
The distributed control of islanded AC microgrids is based on the local measurements of the electrical variables at some nodes of the system to perform the secondary control. The Phasor Measurement Units (PMUs) guarantee accurate synchronized measurements that can be used as input signals for such distributed controllers. This paper analyzes the effect of one of the characteristic parameters of the PMU, the reporting rate, on the control performance. Verifying that could allow the use of low cost measurement devices with the aim of implementing the monitoring and control of low voltage microgrids.
\end{abstract}

Keywords-AC microgrid, Distributed control, PMU, Synchrophasors, Reporting rate

\section{INTRODUCTION}

The control of microgrid is becoming more and more a key topic in the last years, with the results that many voltage controllers have been developed with the purpose of managing the Distributed Generators (DGs) of AC microgrids, such as PVs, wind turbines and electrical storages.

One of the approaches that has received more attention is the hierarchical structure, where a secondary control modifies the set-points of the primary controllers of the DGs in order to restore frequency and voltages of the system when altered following a certain disturbance in the microgrid [1]. One of the methods that has been proposed is the distributed cooperative control, which is based on the graph theory [2][3].

This particular control uses the information from the neighbors, which are other DGs in the microgrid, to control frequency, node voltages, active and reactive powers. The distributed control does not collect all the data in a centralized manner, improving therefore the reliability of the system, given that it requires a complex communication network [3]. Therefore, the distributed approach relies on the communication of data between nodes of the system that are close. Furthermore, some authors have demonstrated that such a controller is able to operate even when there is a reconfiguration in the communication topology [3]. All these features make the distributed control an interesting solution for the AC microgrid.

On the other hand, the research on the Phasor Measurement Units (PMUs) has recently demonstrated that they can be considered a very suitable solution for the distribution network [4][5]. They can be used for protection, monitoring, state estimation and control. In [6], the application of a distributed control based on synchrophasors has been introduced. The authors have focused on the distributed voltage and reactive power control for Medium Voltage system, with a particular attention on the effect of the communication network.

The approach proposed in this paper, instead, focuses on the application of the synchrophasors' measurements for the control of low voltage an islanded AC microgrid. In this sense, the PMUs can play a role in support of the control of the microgrid, given that they can achieve synchronization of the measurement in respect to a common reference in time. Moreover, the PMUs can be placed in different nodes of the system to perform distributed measurements, communicating among them and with the DGs by means of a local communication network [3]. Among the different characteristics of the PMU, a particular attention is paid to the effect of the reporting rate on the distributed cooperative control to demonstrate the applicability of the PMU for this purpose.

The paper is organized as follows: Chapter II describes the islanded AC microgrid, the distributed control and the use of the PMUs for the distributed control, focusing on the difference with the standard PMU and on the impact of the reporting rate. Chapter III shows the results of the simulations and finally, the conclusions are presented in Chapter IV.

\section{PHASOR MEASUREMENTS FOR DISTRIBUTED CONTROL}

\section{A. PMU for Control of AC Microgrids \\ 1) Islanded AC Microgrid and the Primary Control}

The islanded microgrid represents a small portion of a distribution grid, characterized by a limited number of nodes, disconnected from the main grid. Therefore, the main role of the control of the distributed generators is to set the voltage of specific nodes of the systems to the reference values [3]. These nodes are controlled by the secondary control, which is described in Section A2). 


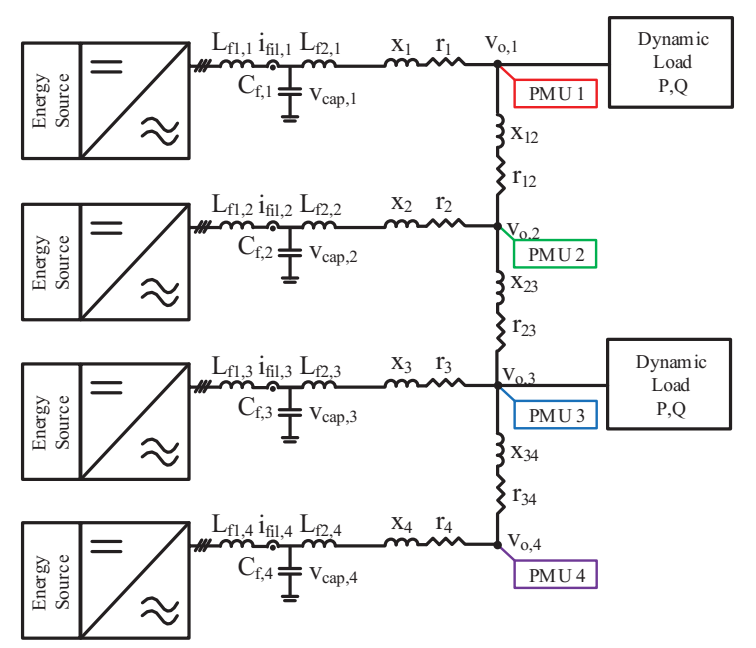

Figure 1: Islanded AC Microgrid

Moreover, every DGs is connected to the low voltage distribution grid by means of a voltage-source inverter (VSI) controlled by the primary control [3][7]. The primary control of the VSI controls the voltage and frequency output of the inverter and it is based on the droop characteristic. The droop equations modify the voltage and frequency reference values [3]:

$$
\begin{gathered}
v_{\text {ref }_{i}}=v_{n_{i}}-n_{i} q_{i} \\
\omega_{\text {ref }_{i}}=\omega_{n_{i}}-m_{i} p_{i}
\end{gathered}
$$

Where $v_{n_{i}}$ is the desired voltage value at the filter capacitance, $q_{i}$ is the output reactive power multiplied by the droop coefficient $n_{i}$ and $p_{i}$ is the output active power multiplied by the droop coefficient $m_{i}$.

The microgrid under test is characterized by four DGs connected to a distribution feeder. Two dynamic loads are connected at two nodes of the microgrid and the PMUs measure the voltage, current and frequency at the four nodes of the microgrid, as described in Figure 1.

\section{2) Secondary Distributed Control of the Microgrid}

This chapter briefly introduces the control strategy that has been tested in the presence of the distributed phasors measurements. The Secondary control has the main role of controlling the voltage and frequency of some of the nodes of the islanded microgrid, which deviate from the desired setpoint. Moreover, the secondary control can perform the power sharing among the different DGs.

The consensus-based distributed control for microgrid has been developed in [3]. The purpose of the control is to reach an agreement space for all the DGs that participate to the voltage control of the microgrid. The control theory is based on the well-known graph theory [2][3], where every node receives the measurement from a certain number of nodes, depending on the graph topology itself. The communication links of the graph, the so-called edges, are represented by the time-invariant adjacency matrix $A[1][3]$, where the element $a_{i j}$ of the matrix defines the weight of the edge from the agent $i$ to $j$.

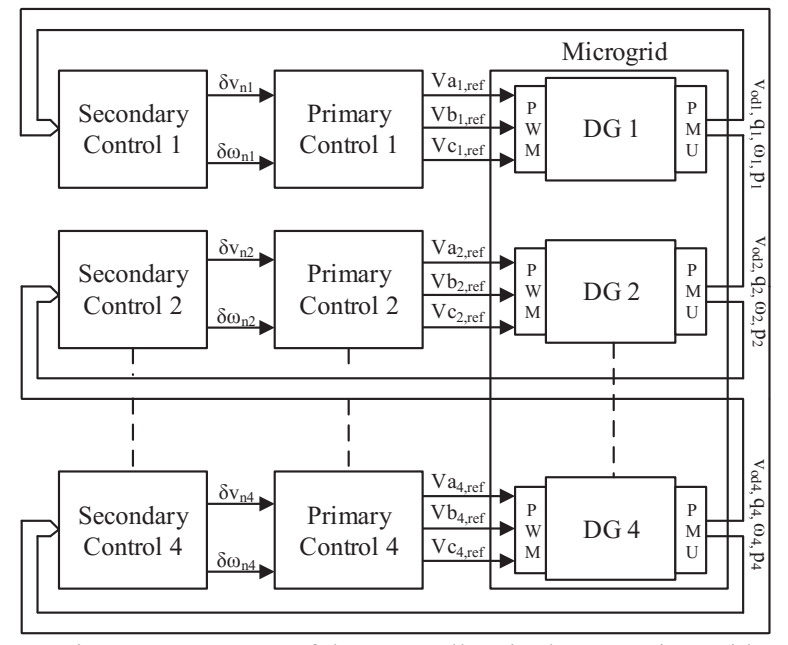

Figure 2: Structure of the Controllers in the AC Microgrid

The control algorithms are defined as four different equations that, combined together, adjust the reference input of the primary control. The equations are defined as follows:

$$
\begin{aligned}
\dot{u}_{v_{i}}= & c_{v_{i}}\left[\sum a_{i j}\left(v_{o d_{j}}(t)-v_{o d_{i}}(t)\right)+\right. \\
& \left.g_{i}\left(V_{\text {ref }}-v_{o d_{i}}(t)\right)\right] \\
\dot{u}_{q_{i}}= & c_{q_{i}}\left[\sum a_{i j}\left(n_{j} q_{j}(t)-n_{i} q_{i}(t)\right)\right] \\
\dot{u}_{\omega_{i}}= & c_{\omega_{i}}\left[\sum a_{i j}\left(\omega_{j}(t)-\omega_{i}(t)\right)+\right. \\
& \left.g_{i}\left(\omega_{r e f}-\omega_{i}(t)\right)\right] \\
\dot{u}_{p_{i}}= & c_{p_{i}} \sum a_{i j}\left(m_{j} p_{j}(t)-m_{i} p_{i}(t)\right)
\end{aligned}
$$

Where the d-component of the node voltages is described by $v_{o d}$ and $\omega$ is the measurement of the frequency at the same node. The coefficients $c_{v_{i}}, c_{q_{i}}, c_{\omega_{i}}, c_{p_{i}}$ govern the convergence speed of the algorithms [3]. The matrix $G=\operatorname{diag}\left\{g_{i}\right\}$ defines the coefficients describing the weight of edges that connect the agents to the references.

The appropriate combination of the output of the secondary control represents the deviation terms that are added to the reference values $v_{n_{i}}$ and $\omega_{n_{i}}$ of the primary control [1], resulting in the followings:

$$
\begin{gathered}
\delta v_{\mathrm{n}_{i}}=K_{I}^{v} \int\left(\dot{u}_{v_{i}}+\dot{u}_{q_{i}}\right) \mathrm{d} t+K_{P}^{v}\left(\dot{u}_{v_{i}}+\dot{u}_{q_{i}}\right) \\
\delta \omega_{\mathrm{n}_{i}}=K_{I}^{\omega} \int\left(\dot{u}_{\omega_{i}}+\dot{u}_{p_{i}}\right) \mathrm{d} t \\
+K_{P}^{\omega}\left(\dot{u}_{\omega_{i}}+\dot{u}_{p_{i}}\right)
\end{gathered}
$$

Figure 2 represents the structure of the control and of the microgrid described in Figure 1. The Secondary control sends the terms $\delta v_{\mathrm{n}_{i}}$ and $\delta \omega_{\mathrm{n}_{i}}$ to the primary control that calculates the three phase reference signals for the PWM calculation.

The controller parameters have been defined by using the controller design guideline described in [10]. Small gain parameters have been used for the secondary control, favoring 
relative stability over faster dynamic response. Moreover, the guideline states that the reactive power control should be slightly slower than voltage controller.

The calculation of the reactive power control can be affected by measurement noise and low reporting rates introduced by the measurement devices [10], reducing therefore the relative stability of the control. The definition of smaller gain parameters $\vec{c}_{q}$ reduces the possibility of reaching some instabilities by slowing down, on the other hand, the converge speed of the reactive power control. The graph topology defines the communication structure that the PMUs use to send the measurements to the secondary controllers as described in Figure 3. The communication channels for each single measurement device are limited to a small number of neighbors. This reduces the complexity of the communication network and improves the reliability of the whole system.

\section{3) Application of PMUs for Distrbuted Control}

The Phasor Measurement Unit, normally measures synchrophasors, frequency and Rate Of Change Of Frequency (ROCOF) as defined in the standard IEEE c37.118-1.2011. The synchrophasors are measured referring to the nominal frequency, that for European power systems is equal to $50 \mathrm{~Hz}$. The phase angle reference is the cosine function that has peak value at the second rollout, where the second time information is provided by the Pulse Per Second (PPS) signal. PMUs are considered to be devices relevant for monitoring application but also can be exploited for real time control functions, since many of them leverage on absolute phase angle information from nodes located at different geographical locations of the grid as well as accurate time tags.

The distributed cooperative control strategy, described in section A2), has been selected for its dependency on the phase measurement, given that it uses the dq-transformed measurement of the voltage, as depicted in Figure 1. In the transformation, the measurement of the phase of the voltage obtained from the PMU is used to align the dq rotating reference frame to the $\mathrm{d}$ component. The synchronized phase measurements of different nodes of the microgrid allow the comparison of the d components because they are referred to a common reference. Since the PMU calculates the phasors of the voltages and currents, they can be also used for the calculation of the active and reactive power generated by each DG.

Therefore, the control is clearly subjected to the effect of some errors on the phase measurement, related to reporting rate, synchronization errors and to the transducer phase displacement [8]. The paper focuses on the evaluation of the limitation introduced by the reporting rate on the control performance, neglecting other factors that can degrade the phase measurement. The synchronized phase information given by the PMUs could be eventually used in case of a disconnected inverter that has to be synchronized to the phase of the grid before being reconnected. Similarly, the information on the phase can be used in the reconnection procedure of the islanded microgrid to the main grid [11].

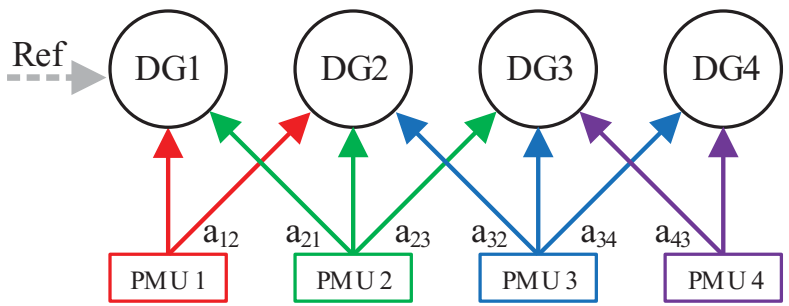

Figure 3: Communication structure of the graph

In all these situations, a small difference in the phase angle can introduce high current transient peaks [11]. This demonstrates how the synchronized phase measurement can have a positive impact on the management of the microgrid.

Eventually, the PMU is a suitable measurement device for the distributed control strategy, given that the communication interfaces and protocols have been already standardized for substation automation [12]. In the aforementioned control strategy, the communication paths follow the graph structure, where every edge represents the channel between the transmitter, the PMU at a specific node, and the receiver, which is one of the controllers, as shown in Figure 2 and Figure 3.

\section{4) Effect of the Reporting rates}

The effect of the measurement of the PMU on the control performance has been tested versus diverse update frequencies. The reporting rate, measured in frames/sec, represents a critical aspect of the distributed control.The reporting rate represents also the frequency at which the control output is updated. The controller receives the information of a certain disturbance in the system, such as a load change, from the measurements and consequently adapts the control output to that. Therefore, the reduction of the update frequencies can reduce the ability of the controller to react to a disturbance in the system.

\section{5) Difference from standard PMUs}

In order to adapt the PMU measurements to the aforementioned control applications, some considerations have to be done.

The standard IEEE c37.118.1-2011 requires to refer the phase angle with respect to a $50 \mathrm{~Hz}$ cosine function. This means having a rotating phasor with equivalent frequency equal to the difference between the actual system frequency and the nominal system frequency. For instance, in case the system has a frequency of $50.1 \mathrm{~Hz}$, the equivalent phasor will do a rotatation of $360^{\circ}$ every 10 seconds.

The aforementioned control algorithm, however, relies on a fixed phase angle information, that should be referred to a cosine function with frequency equal to the current system frequency. Such features is obtained, by applying a compensation of the phase angle information as defined in

$$
\Delta \alpha=\Delta f \cdot T
$$




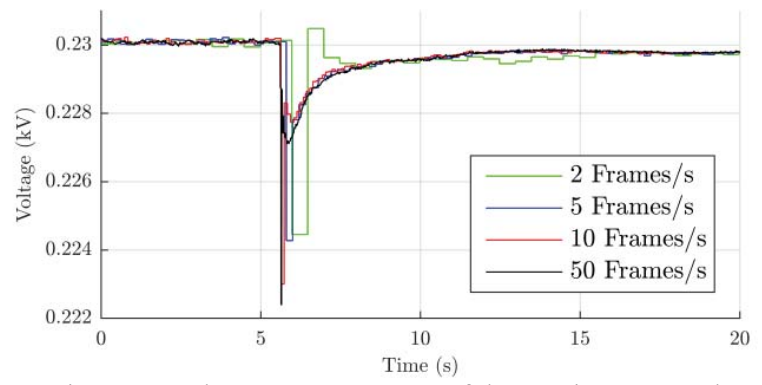

Figure 4: Voltage measurements of the maximum overshoot for different reporting rates

Where $\Delta f$ is the frequency deviation from the nominal frequency $(50 \mathrm{~Hz})$ and $\mathrm{T}$ is the update rate of the PMU (e.g. 20 $\mathrm{ms})$. Moreover, the current standard IEEE c37.118.1-2011, envision the communication architecture as strictly hierarchical, with PMUs pushing measurements to the socalled Phasor Data Concentrators (PDCs), which in turn, can forward the measurements to Super PDCs.

In this paper, it is also considered the possibility to have horizontal information exchange, that is PMU to PMU. Each unit has, therefore, server and client installed, in order to publish synchrophasors and at the same time subscribe to phasors published by neighbor nodes. Given that the PMU sends measurements only to the neighbor nodes, the overall communication traffic is still limited.

\section{Simulations Results}

In the simulation tests, the effect of the reporting rate has been studied as a first step in the analysis of the PMUs for distributed control of low voltage microgrids. The analysis of other factors that can affect the control performance have been intentionally omitted.

The simulation tests have been performed by means of the Real Time Digital Simulator (RTDS), where the complete microgrid, the controllers and the PMUs have been modeled. The time-step of the simulation is $T_{s}=100 \mu \mathrm{s}$, whereas the inverters are simulated with a small time step that can vary between 1.4 and $2.5 \mu$ s to simulate the switching.

The PMU, in the modelling environment of RTDS, includes the algorithm for calculation of synchrophasors that handles the digital samples of the simulation. The algotihm matches the accuracy requirements for steady state and dynamic state conditions for the $\mathrm{P}$ (protection) class of the IEEE c37.118.1-2011 standard. It includes a hamming window function, a low pass digital Finite Impulse Filter (FIR) filter, the calculation of the $50 \mathrm{~Hz}$ components of magnitude and phase angles of voltage and current phasors of the currents and the calculation of the frequency. Power measurements are also inferred, by means of the voltage and currents measured by the PMU block.

The updating frequency varies from a high value, quite difficult to reach for measurement devices applied to distribution network to a low rate, which can be obtained with low cost devices. The reporting rates that have been selected from the PMU block are 50, 10, 5, 2 frames/s, which are all sub-multiples of the nominal frequency [9].

Table I: Parameters of the Secondary Control

\begin{tabular}{ccccc}
\hline & DG1 & DG2 & DG3 & DG4 \\
\hline $\boldsymbol{v}_{\boldsymbol{n}_{\boldsymbol{i}}}(\boldsymbol{V})$ & 380 & 380 & 380 & 380 \\
\hline $\begin{array}{c}\boldsymbol{\omega}_{\boldsymbol{n}_{\boldsymbol{i}}} \\
\boldsymbol{r a d} / \boldsymbol{s})\end{array}$ & $2 \pi \cdot 50$ & $2 \pi \cdot 50$ & $2 \pi \cdot 50$ & $2 \pi \cdot 50$ \\
\hline $\boldsymbol{P}_{\boldsymbol{r} \boldsymbol{e f _ { \boldsymbol { i } }}}(\boldsymbol{W})$ & $6 \cdot 10^{3}$ & $6 \cdot 10^{3}$ & $6 \cdot 10^{3}$ & $6 \cdot 10^{3}$ \\
\hline $\boldsymbol{Q}_{\boldsymbol{r} \boldsymbol{e}_{\boldsymbol{i}}}(\boldsymbol{V} \boldsymbol{A R})$ & $1 \cdot 10^{3}$ & $1 \cdot 10^{3}$ & $1 \cdot 10^{3}$ & $1 \cdot 10^{3}$ \\
\hline $\boldsymbol{m}_{\boldsymbol{i}}$ & $7 \cdot 10^{-6}$ & $5 \cdot 10^{-6}$ & $5 \cdot 10^{-6}$ & $7 \cdot 10^{-6}$ \\
\hline $\boldsymbol{n}_{\boldsymbol{i}}$ & $2 \cdot 10^{-4}$ & $3 \cdot 10^{-4}$ & $3 \cdot 10^{-4}$ & $2 \cdot 10^{-4}$ \\
\hline $\boldsymbol{K}_{\boldsymbol{I}}^{\boldsymbol{v}}, \boldsymbol{K}_{\boldsymbol{P}}^{\boldsymbol{v}}$ & 0.33, & 0.33, & 0.33, & 0.33, \\
\hline $\boldsymbol{K}_{\boldsymbol{I}}^{\boldsymbol{\omega}}, \boldsymbol{K}_{\boldsymbol{P}}^{\boldsymbol{\omega}}$ & $5 \cdot 10^{-2}$ & $5 \cdot 10^{-2}$ & $5 \cdot 10^{-2}$ & $5 \cdot 10^{-2}$ \\
\hline $\boldsymbol{c}_{\boldsymbol{v}}, \boldsymbol{c}_{\boldsymbol{q}}, \boldsymbol{c}_{\boldsymbol{q}}^{\mathbf{2 H z}}$ & 0.25, & 0.25, & 0.25, & 0.25 \\
\hline $\boldsymbol{c}_{\boldsymbol{\omega}}, \boldsymbol{c}_{\boldsymbol{p}}$ & $1,0.1,0.04$ & $1,0.1,0.04$ & $1,0.1,0.04$ & $1,0.1,0.04$ \\
\hline & 1,1 & 1,1 & 1,1 & 1,1 \\
\hline
\end{tabular}

The measurements are assumed to be received by the controllers with delay equal to zero, because of the same simulation framework. Moreover, since the aim of the paper is to evaluate the effect of the reporting rate, no additional delays have been introduced.

The reporting rate represents the only element that significantly deteriorate the quality of the measurement of the simulations, given that the PMU block does not introduce synchronization errors and the transducers has been not considered

The tests consist on performing load step variations on the two loads performed five hundred times for each reporting rate. The duration of the real time simulation is 60 seconds, in order to wait for the controllers to react and stabilize the system to the new steady state values. After this time, the level of the loads is set to the initial conditions to start a new load step, for a total number of 500 simulations. Each simulation test starts with a base power of $5 \mathrm{~kW}$ and $1 \mathrm{kVAR}$ for each load. Therefore, the load power is changed with a step of $5 \mathrm{~kW}+/$ $10 \%$ and $0.5 \mathrm{kVAR}+/-10 \%$. The load variation is performed at time $\mathrm{t}=6 \mathrm{~s}$ for the first dynamic load, whereas the second load varies the power randomly within a second after the first load step.

The values of the control parameters are described in Table I. As it is described in the Table I, for the reporting rate 2 Frames/s the coefficient $\boldsymbol{c}_{\boldsymbol{q}}$ has been reduced to maintain the stability, given that the coefficient used for the other reporting rates introduced instabilities.

Table II: Undershoot of Voltage and Frequency

\begin{tabular}{ccccccccc}
\hline & \multicolumn{2}{c}{$\mathbf{5 0} \mathbf{~ H z}$} & \multicolumn{1}{c}{$\mathbf{1 0} \mathbf{~ H z}$} & \multicolumn{2}{c}{$\mathbf{~ H z}$} & \multicolumn{2}{c}{$\mathbf{2 ~ H z}$} \\
\hline & $\mathbf{V}$ & $\mathbf{f}$ & $\mathbf{V}$ & $\mathbf{f}$ & $\mathbf{V}$ & $\mathbf{f}$ & $\mathbf{V}$ & $\mathbf{f}$ \\
\hline $\begin{array}{c}\text { Max } \\
(\%)\end{array}$ & 3.31 & 0.38 & 3.04 & 0.40 & 2.48 & 0.27 & 2.40 & 0.25 \\
\hline $\begin{array}{c}\text { Mean } \\
(\%)\end{array}$ & 1.91 & 0.21 & 1.37 & 0.08 & 1.31 & 0.05 & 1.35 & 0.02 \\
\hline $\begin{array}{c}\text { Std. } \\
\text { Dev. }\end{array}$ & 0.25 & 0.04 & 0.36 & 0.08 & 0.28 & 0.07 & 0.25 & 0.04 \\
\hline
\end{tabular}




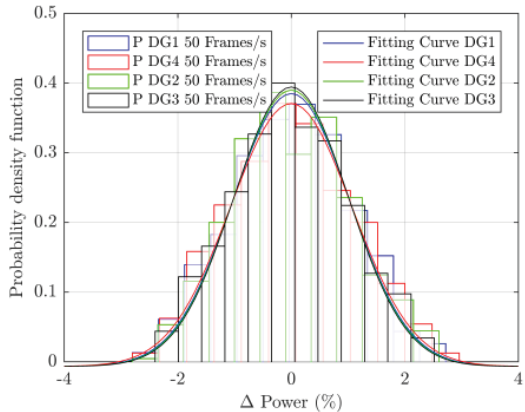

Figure 5: Histogram Active Power 50 Frames/s

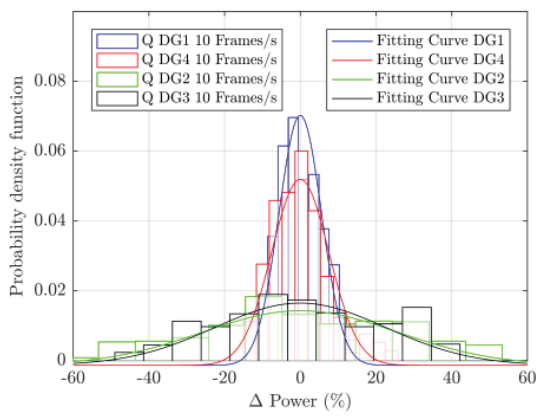

Figure 8: Histogram Reactive Power 10 Frames/s

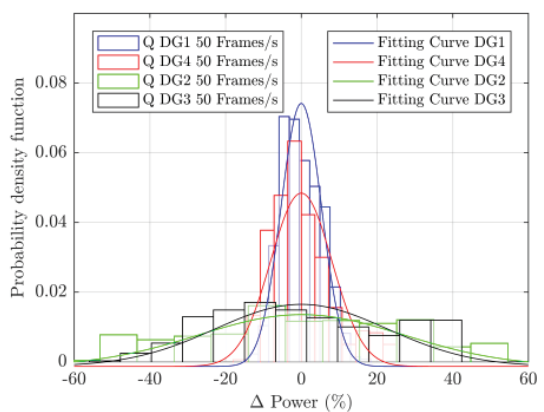

Figure 6: Histogram Reactive Power 50 Frames/s

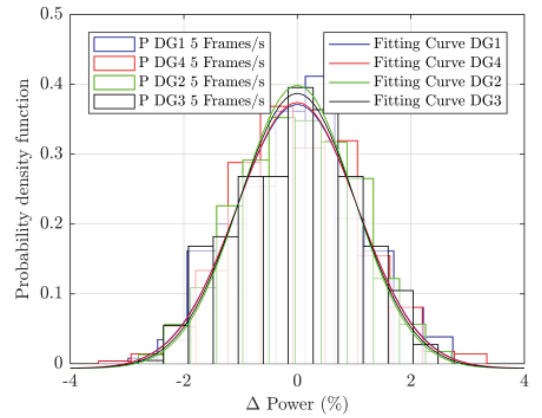

Figure 9: Histogram Active Power 5 Frames/s

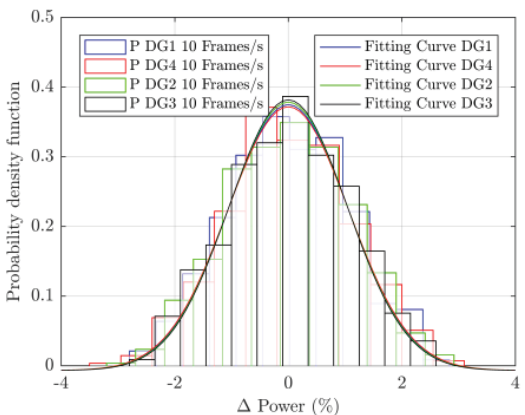

Figure 7: Histogram Active Power 10 Frames/s

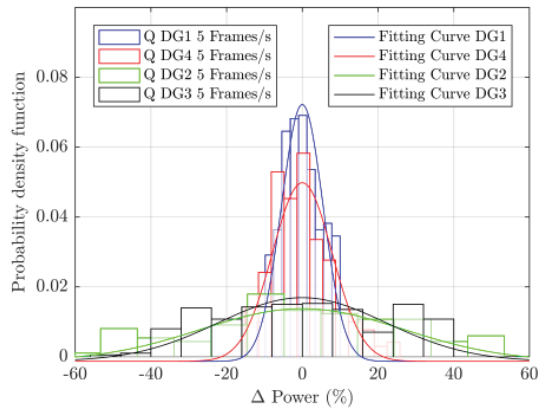

Figure 10: Histogram Reactive Power 5 Frames/s

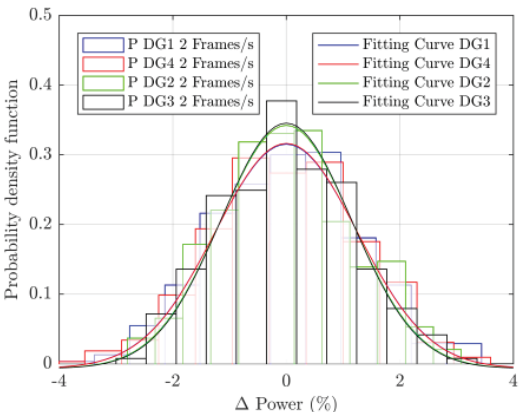

Figure 11: Histogram Active Power 2 Frames/s

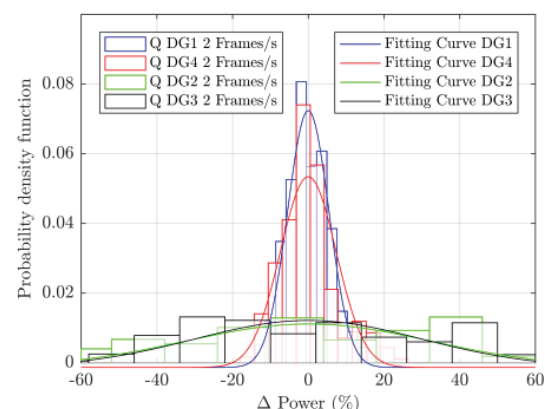

Figure 12: Histogram rective Power 2 Frames/s
The results of the simulation tests have been analyzed in term of voltage and frequency undershoot and in term of percent distribution of the generated powers.

Table II reports the maximum, mean value and standard deviation of the percentage undershoot with respect to the reference values. The analysis is performed on Node 1 , which is the one that it is controlled with a reference value, as described in Figure 3. Table II demonstrates that the reduction of the reporting rate does not affect significantly the action of the voltage and frequency control. The clear difference between the maximum value and the mean value of the overshoot demonstrates that, in most of the cases, the lower reporting rates are not able to detect fast frequency overshoots. The Figure 4 shows the effect of the different reporting rates on the voltage at Node 1, expressed in d component. The figure shows the maximum overshoot test cases, demonstrating that the reduction of the number of updated measurements per seconds does not significantly affect the quality of the control in term of undershoot and in term of the time to reach the steady-state. The design of the secondary control has been performed in such a way that the time to reach the agreement is longer than the minimum measurement update rate. This time separation has been realized by defining an appropriate time constant for the PI controllers and reducing the value of the convergence speed coefficient of the reactive power control. Figure 4 underlines that the decreasing of the reporting rate introduces a delay on reacting to the voltage drop, given that the controller must wait for the next update of the voltage 
measurement before changing the references of the primary controllers.

The second analysis takes into account the quality of the power sharing. For a specific reporting rate and for each single simulation, the mean values of the measurements of the active and reactive power generated by the DGs after the transient have been calculated. The values have been divided by the calculation of their total mean value. Figure 5-12 show the histograms of the probability density function for the different reporting rates. The horizontal axis represents the percentage variation of the generated active and reactive powers referred to the calculated overall mean value. The histograms show a quasi-gaussian distribution, therefore the fit with a gaussian curve has been calculated.

The histograms of the active power (Figure 5, Figure 7, Figure 9, Figure 11) show a distribution that for all the four cases is between $+/-4 \%$ of the overall mean values. For every single reporting rate, the generated active powers exhibit almost the same fit, showing that the controller governs the sharing with the same dynamic. The test with reporting rate 2 frames/s shows a small increase of the width of the distribution without considerably decreasing the quality of the power sharing. The histograms of Figure 6, Figure 8, Figure 10, Figure 12 exhibit a wider distribution, demonstrating that the control of the reactive power is more affected by errors on the power sharing related to the errors on the measurement. The figures show that there is a clear difference between the distribution of the reactive power of the DG1 and DG4, in blue and red respectively, and of the DG2 and DG3, green and black. The former group possesses a smaller value of the droop coefficient, generating more reactive power, while the latter generate less reactive power because of the smaller droop coefficient. Figure 12 shows that the reduction of the coefficient $c_{q}$ for the reporting rate 2 frames/s, described in Table I, mostly impacts the probability distribution of the DG2 and DG3. The results evidence that sharing of the reactive power is overall more affected by the reporting rate, which can be mostly related to the impossibility of achieving proportional reactive power sharing and accurate voltage regulation with the proposed distributed control [13]. In this paper has been chosen to privilege voltage control rather than reactive power sharing. Moreover, the method used for the calculation of the reactive power suffers from non-sinusoidal condition [14].

\section{CONCLUSIONS}

The paper has demonstrated the application of synchrophasor measurements for the control of an islanded AC microgrid. The effect of the reporting rate, measured in frames/s, on the quality of the voltage and frequency control and on the active and reactive power sharing control of the distributed cooperative secondary control has been evaluated. The analysis has demonstrated that the reduction of the reporting rate does not reduce the quality of the voltage and frequency control in term of undershoot caused by a load change. The active and reactive generated powers are also not significantly subjected to the effect of reducing the reporting rate, although the reactive power exhibit generally larger errors compared to the active power. This aspect will be deeply investigated in the next steps, by testing the effect of different techniques for the control of the reactive power applied to decentralized control.

The simulation tests have not focused on the use of the synchronized measurement for the connection of DGs to the microgrid and for the reconnection of the microgrid to main grid, even though, as explained in section II.A3), the PMUs can play a significant role on improving these procedures.

The intention of the authors is to proceed with the analysis of the additional features of the PMU and test the complete control and PMU measurements of the microgrid during islanded mode and reconnection procedure in Hardware in the Loop environment.

\section{ACKNOWLEDGMENT}

This work is sponsored by the German Ministry for Industry and Energy (project number 03ET7549A) and the European Union's Horizon 2020 Research and Innovation Programme (Grant Agreement number 700416).

\section{REFERENCES}

[1] Bidram, Ali, and Ali Davoudi. "Hierarchical structure of microgrids control system." IEEE Transactions on Smart Grid 3.4 (2012): 19631976.

[2] Olfati-Saber, Reza, J. Alex Fax, and Richard M. Murray. "Consensus and cooperation in networked multi-agent systems." Proceedings of the IEEE 95.1 (2007): 215-233.

[3] Bidram, Ali, et al. "Secondary control of microgrids based on distributed cooperative control of multi-agent systems." IET Generation, Transmission \& Distribution 7.8 (2013): 822-831.

[4] J. Sexauer, P. et al. "Phasor measurement units for the distribution grid: Necessity and benefits," 2013 IEEE PES Innovative Smart Grid Technologies Conference (ISGT), Washington, DC, 2013, pp. 1-6.

[5] Seyedi, Younes, and Houshang Karimi. "Coordinated Protection and Control Based on Synchrophasor Data Processing in Smart Distribution Networks." IEEE Transactions on Power Systems (2017).

[6] Borghetti, Alberto, et al. "Synchrophasors-Based Distributed Secondary Voltage/VAR Control via Cellular Network." IEEE Transactions on Smart Grid 8.1 (2017): 262-274.

[7] Rocabert, Joan, et al. "Control of power converters in AC microgrids" IEEE transactions on power electronics 27.11 (2012): 4734-4749.

[8] Monti, A., C. Muscas, and F. Ponci. "Phasor Measurement Units and Wide Area Monitoring Systems." (2016).

[9] IEEE Standard for Synchrophasor Measurements for Power Systems," in IEEE Std C37.118.1-2011 (Revision of IEEE Std C37.118-2005), vol., no., pp.1-61, Dec. 282011

[10] Nasirian, Vahidreza, et al. "Droop-free distributed control for AC microgrids." IEEE Transactions on Power Electronics 31.2 (2016): 16001617.

[11] D. Shi, Y. Luo and R. K. Sharma, "Active synchronization control for microgrid reconnection after islanding," IEEE PES Innovative Smart Grid Technologies, Europe, Istanbul, 2014, pp. 1-6.

[12] IEC TR 61850-90-5:2012 - Use of IEC 61850 to transmit synchrophasor information according to IEEE C37.118

[13] Han, Yang, et al. "Review of active and reactive power sharing strategies in hierarchical controlled microgrids." IEEE Transactions on Power Electronics 32.3 (2017): 2427-2451.

[14] Cataliotti, Antonio, et al. "The measurement of reactive energy in polluted distribution power systems: An analysis of the performance of commercial static meters." IEEE Transactions on Power Delivery 23.3 (2008): 1296-1301. 